\title{
ChemComm
}

CrossMark <click for updates

Cite this: Chem. Commun., 2015, 51, 11884

Received 4th June 2015

Accepted 22nd June 2015

DOI: $10.1039 / \mathrm{c5cc04602e}$

www.rsc.org/chemcomm

\section{Biomimetic self-assembling acylphthalocyanines $\dagger$}

\author{
Hong-Guang Jin, Mihaela Carmen Balaban, Sabine Chevallier-Michaud, \\ Michel Righezza and Teodor Silviu Balaban*
}

\begin{abstract}
We synthesized a series of biomimetic self-assembling phthalocyanines equipped with carbonyl groups as recognition motifs, a central zinc atom and diverse solubilizing alkyl chains mimicking for the first time with these robust pigments the natural chlorosomal bacteriochlorophylls. Upon self-assembly a very broad and red-shifted Q-band absorption extending to over $900 \mathrm{~nm}$ is put into evidence.
\end{abstract}

Highly ordered self-assembling functional dyes with unique photoelectric properties as a consequence of excitonic interactions between neighboring dye monomers are desirable for materials science. ${ }^{1} \mathrm{~A}$ well-defined manner connecting these dye monomers are crucial for a device to transfer the excitation energy to a designated acceptor for efficient energy utilization. ${ }^{2}$ Compared to traditional covalent bonds, the supramolecular self-assembly based on noncovalent interactions is an useful approach to reach this goal, including (i) relative easy synthesis of monomers, (ii) the self-healing and thermodynamically stable self-assemblies. Phthalocyanines $(\mathrm{Pc}$ 's $),{ }^{3}$ are the most stable and robust light-harvesting system with high absorption coefficients in the visible and near-infrared region. However, Pc's based devices have been rather limited due to their poor solubility in common organic solvents and their disordered aggregation on the corresponding surface, ${ }^{4}$ meanwhile, in contrast with other dyes, ${ }^{5}$ incorporating specific recognition sites into Pc's to induce self-assembly, is still synthetically challenging.

Natural light-harvesting systems function with high efficiencies, also under low-light illumination conditions. Green photosynthetic bacteria can scavenge photons even at one hundred meters under water surface ${ }^{6}$ thanks to their special organelles, called chlorosomes, which agglomerate homologues of bacteriochlorophylls (BChl's) $c, d$, and $e$ as functional pigments (Fig. 1a). In polar solvents, the monomeric forms prevail having an intense and

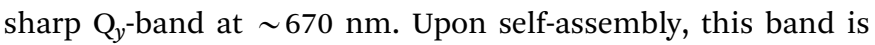

Aix-Marseille Université, CNRS UMR 7313, Centrale Marseille, Chirosciences, Service 442, Avenue Escadrille Normandie-Niemen, F-13013 Marseille, France. E-mail: ts.balaban@univ-amu.fr

$\dagger$ Electronic supplementary information (ESI) available: Synthetic procedures, product characterization, spectral data. See DOI: 10.1039/c5cc04602e red-shifted to $\sim 740 \mathrm{~nm}$ and increases by up five times its full-width-at-half-maximum mainly due to inhomogeneous broadening. ${ }^{7}$ Our laboratory has mimicked $^{8}$ and extended the self-assembly algorithm ${ }^{9}$ (Fig. 1b) by employing different recognition motifs as well as various solubilizing groups grafted onto porphyrins or chlorins which self-assemble in the same way as the natural BChl's. We report here on equipping Pc's with acyl recognition groups which will then induce self-assembly under appropriate conditions in a similar manner as the natural BChl's and our previously reported 3,13-diacetyl-Zn-porphyrins for which by means of single crystal X-ray structures we could unravel their supramolecular architecture, essential for light-harvesting. ${ }^{10}$

Herein we present a series of self-assembling tetra-acylated ZnPc's with long alkyl chains as solubilizing groups and carbonyl groups as the recognition groups mimicking the self-assembly algorithm of BChl's. When the self-assembly operates, a broad a)

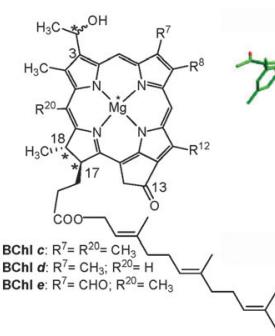

c)

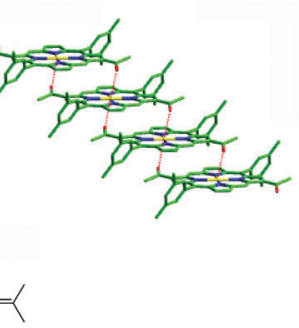

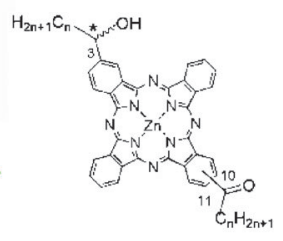

Fig. 1 (a) Natural self-assembling BChl's where $\mathrm{R}^{8}$ and $\mathrm{R}^{12}$ can be homologous groups such as methyl, ethyl, $n$-propyl or isobutyl. Farnesol is depicted as the long-chain alcohol esterifying the 17-propionic acid but also other fatty alcohols such as stearol, cetol, phytol, geranyl-geraniol, etc. can be encountered in various bacteria. Asterisks denote stereocenters. (b) Single crystal structure of a BChl mimic: 3,13-diacetylporphyrin having Zn

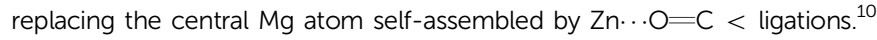
A tetramer is shown with the partially disordered 3,5-di-tert-butyl groups truncated at their ipso carbon for clarity. Broad and red-shifted absorption bands characterize this stair-case-like architecture, typical for J-aggregates. ${ }^{11}$ (c) Targeted constructs in this work whereby a diacylated ZnPc is statistically monoreduced to a ketol with alkyl chains of various lengths for tuning the solubility. 
and red-shift near-infrared absorption onset at over $900 \mathrm{~nm}$ can be seen in the electronic absorption spectra. The temperature dependence of the equilibrium between the monomer and the aggregated species was also studied.

Prior to our studies, ${ }^{12}$ no acyl-Pc's had been described in the literature. This is probably due to the reactivity of the carbonyl groups under the strongly basic and high temperature conditions required for the templated cyclo-tetramerization by divalent metal salts of the Pc synthons. In a shot-gun approach we tried in vain to acylate under various Friedel-Crafts conditions the unsubstituted CuPc. Due to its insolubility in various solvents, unreacted material was always recovered. In concentrated sulfuric acid, apparently a good solvent for $\mathrm{CuPc}$, neither anhydrides nor acyl chlorides reacted with or without preformed complexes of $\mathrm{AlCl}_{3}$.

We then turned our attention to a multistep synthetic sequence which was optimized as shown in Scheme 1. Friedel-Crafts acylation of ortho-dichlorobenzene, which is available in bulk quantities, with various acyl chlorides, also available on an industrial scale, provided the 4-acyl-1,2-dichlorobenzene derivatives $2 \mathrm{a}$-d with $\mathrm{R}=\mathrm{C}_{n} \mathrm{H}_{2 n+1}$ $(n=1,5,11,15)$. Direct conversion to the phthalodinitriles occurred unproblematically by Pd-catalysis but the cyclotetramerization reaction to the corresponding Pc's, although it occurred in good yields, was accompanied by a Meerwein-Pondorf-Verley type reaction. Thereby some of the carbonyl groups became reduced to the secondary alcohols, giving much too complex reaction mixtures, albeit their self-assembly could be undoubtedly proven. ${ }^{12}$

Therefore we restored to protect at the early 4-acyl-1,2dichlorobenzene stage the carbonyl groups with neopentyl glycol, which can eventually be easily removed. Cyanation involving palladium catalysis of these derivatives led to the precursor phthalodinitriles $\mathbf{4 a - d}$ in moderate to high yields. Their cyclotetramerization gave the tetracarbonyl-protected ZnPc-5a-d.

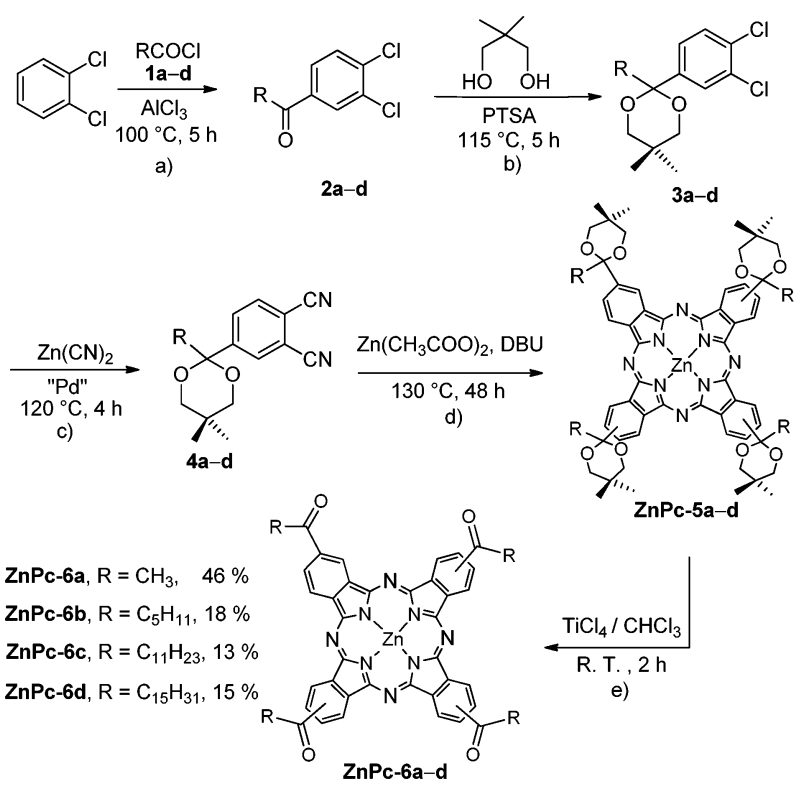

Scheme 1 Synthetic route of the desired tetraacylated ZnPc-6a-d. Yields for procedures (a)-(c) performed several times on a multigram scale are detailed in the ESI. $\dagger$ The overall yields for $\mathbf{Z n P c - 6 a - d}$ are based on the procedures (d) and (e) having $4 a-d$ as starting material.
Finally, deprotection of the carbonyl groups proved to be possible with $\mathrm{TiCl}_{4}$ furnishing the desired tetraacylated ZnPc-6a-d. The synthetic intermediates, excluding $\mathbf{2 a}$, are also novel compounds which could have interesting applications as they can be easily obtained cheaply in large quantities.

After succeeding in equipping the series of ZnPc-6a-d with carbonyl groups and a central zinc atom as recognition groups, we then performed self-assembly experiments of these compounds in nonpolar solvents. With the increasing solubility brought about by the longer alkyl chains, the ZnPc-6b-d could self-assemble in solvents of low polarity such as $n$-heptane, while ZnPc-6a could also self-assemble in moderately polar solvents such as dichloromethane and chloroform (Fig. S3, ESI $\dagger$ ). Below, we present ZnPc-6d as an example of the self-assembly process.

We performed UV-Vis absorption measurements in dry $n$-heptane at room temperature for ZnPc-6d (Fig. 2). The pure sample powder and the cuvettes were thoroughly vacuum dried and the prepared solution was also sonicated for 2 min before the measurement. From the absorption spectra, a very broad and redshifted Q-band absorption with an onset at over $900 \mathrm{~nm}$ could be seen, which is rare among the phthalocyanines. When two drops of methanol were added to the $3 \mathrm{~mL}$ cuvette, only a partial disassembly occurred because methanol can ligate and compete to the coordination of the central zinc ion. The Pc assemblies are thus much more robust than our previous porphyrinic BChl mimics where stoichiometric amounts of Zn-coordinating alcohols or even adventitious water were sufficient to monomerize the aggregates. ${ }^{8,9}$ Further additions of five drops of methanol led to phase separation which could be prevented by further adding five drops of dichloromethane thus ensuring a homogeneous solution. Thereby, the aggregates disassemble completely, giving intense monomeric Q-bands, with no aggregates absorbing above $750 \mathrm{~nm}$.

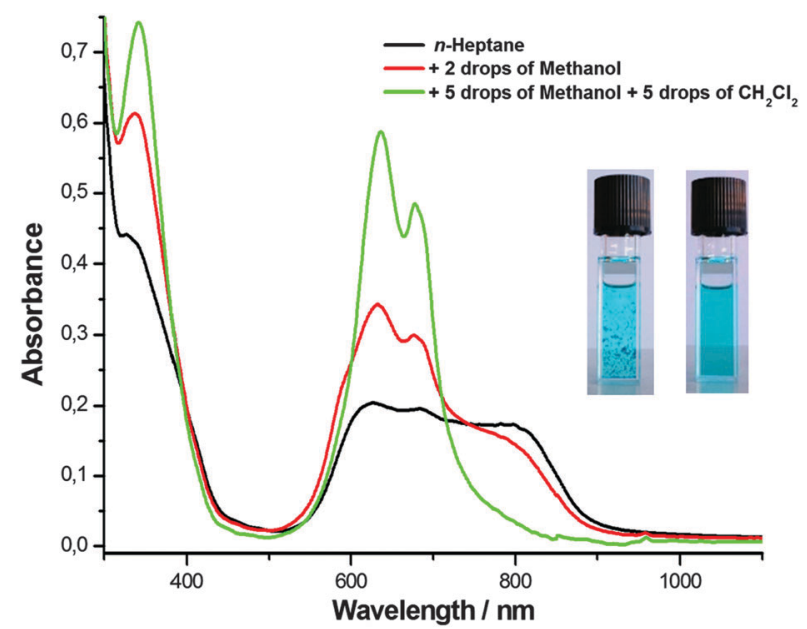

Fig. 2 Absorption spectra of ZnPc-6d in dry $n$-heptane $\left(3.3 \times 10^{-5} \mathrm{M}\right.$, black line), after addition of two drops of methanol (red line) and followed by five more drops of methanol and five drops dichloromethane (the green line). A $3 \mathrm{~mL}$ cuvette with $1 \mathrm{~cm}$ pathlength is shown before (at left) and after addition of methanol and gentle shaking (at right). A microscopic image of the fluffs characteristic of these macro aggregates is provided in the ESI $\dagger$ (Fig. S7). Instead of methanol, pyridine or THF can be used to disassemble the aggregates (Fig. S9, ESI†). 
Noteworthy is that during the disassembly process, the absorption bands and fluorescent emission attributed to the monomer were also intensified gradually (Fig. S6, ESI $\dagger$ ). Meanwhile, in the absence of Zn-coordinating solvents, slow growth of aggregates from a few hundred nanometres to visible fluffs which flocculate in the $n$-heptane solution could also be seen by naked eye or microscopy (Fig. S7, ESI $\dagger$ ). Gentle shaking or short sonication restores homogeneous solutions which were stable for various spectroscopic studies.

All of the above phenomena resembled both that of the natural BChl's and of our previous mimics. In order to further study the self-assembly and disassembly process, variable-temperature UV-Vis absorption measurements were performed from $T=20$ to $95{ }^{\circ} \mathrm{C}$. As shown in Fig. 3, an equilibrium could be put into evidence. During the temperature-rise period, the aggregates gradually disassemble to monomers while in the temperature-fall period, the monomers again self-assemble almost fully reversibly, to the aggregates. Upon heating the aggregates gradually disassemble in a fashion resembling the melting of DNA with a monotonous variation (red curves in the insets of Fig. 3). Evidence for a cooperative and autocatalytic aggregate growth process is provided by the sigmoidal shape of the absorbance upon cooling (blue traces in the insets of Fig. 3). These show an inflexion point at $65{ }^{\circ} \mathrm{C}$ (thin black lines) at both monitored wavelengths. Very similar trends were earlier encountered for the natural $\mathrm{BChl} c$, where a detailed kinetic modeling provided the size of a critical nucleus of about 14 molecules. ${ }^{13}$ Assemblies larger than this critical nucleus grow to macroscopic sizes, while smaller oligomers disassemble.

In order to verify that the biomimetic self-assembly is indeed operating, we used the same conditions for diluting into dry $n$-heptane the carbonyl protected compound ZnPc-5d. In this case only monomeric absorption spectra were observed with no

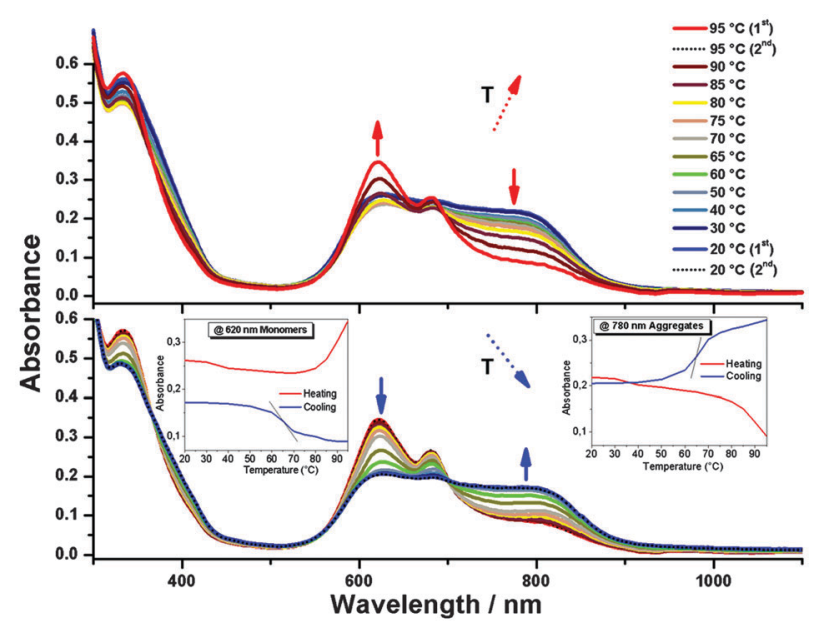

Fig. 3 Temperature-variable UV-Vis absorption of ZnPc-6d $\left(3.3 \times 10^{-5} \mathrm{M}\right)$ in dry $n$-heptane containing $\sim 2 \%$ dry dichloromethane. Above is the temperaturerise period and below is the temperature-fall period. The solid arrows reflect the electronic absorption's trend. Insets show the variable temperature evolution of the aggregate maxima monitored at $780 \mathrm{~nm}$ and that of the monomeric building blocks monitored at $620 \mathrm{~nm}$. Black dotted traces show the almost perfect reversibility of the absorbance after a heating/cooling cycle. red-shifts or band broadening (Fig. S8, ESI $\dagger$ ). However, the ketals ZnPc-5, due to steric hindrance, prevent the formation of face-to-face $\mathrm{H}$-aggregates ${ }^{11 d, e}$ which could translate into the blueshifted shoulders and peaks of the Q-bands encountered in the spectra of the deprotected ZnPc-6 after addition of Zn-coordinating solvents such as methanol, THF or pyridine (Fig. S9, ESI $\dagger$ ).

High-performance liquid chromatography (HPLC) optimizations have been performed on different columns in order to achieve baseline separation of the regioisomers of ZnPc-5a-d and ZnPc-6a-d. Since the pioneering studies of Michael Hannack $^{14}$ and Clifford Leznoff, ${ }^{15}$ very few authors report efficient resolutions of such regioisomers and usually a statistical mixture is implicitly assumed when a nonsymmetrically substituted phthalodinitrile is cyclotetramerized. In our hands, we often encountered serious deviations from the 50\%, 25\%, 12.5\% and $12.5 \%$ for our $C_{\mathrm{s}}, C_{2 \mathrm{v}}, C_{4 \mathrm{~h}}$, and $D_{2 \mathrm{~h}}$ regioisomers, respectively (A $\mathbf{A}_{\mathbf{4}}$ in Scheme 2).

We did had previous experience with reverse phase HPLC separations of similarly substituted water-soluble Pc's. ${ }^{16}$ An elegant study of water soluble Pc's, with a view on photodynamic therapy applications, stands out by the very careful experimentations and the efficient HPLC separations. ${ }^{17}$

Even more complex mixtures resulted in our hands when the protected phthalodinitriles $\mathbf{4 a}-\mathbf{d}$ (A) were submitted to a mixed condensation with the unsubstituted phthalodinitrile (B in Scheme 2). In these cases, beside the four regioisomers $\mathbf{A}_{\mathbf{4}}$ of type ZnPc-5, highly colored Pc's having one, two, or three dioxolane (D) protected acyl residues were formed, beside some unsubstituted $\mathbf{Z n P c}\left(\mathbf{B}_{\mathbf{4}}\right.$ in Scheme 2 ) which could be easily separated due to its limited solubility. HPLC coupled with massspectrometric and diode-array UV-Vis detectors allow analytical
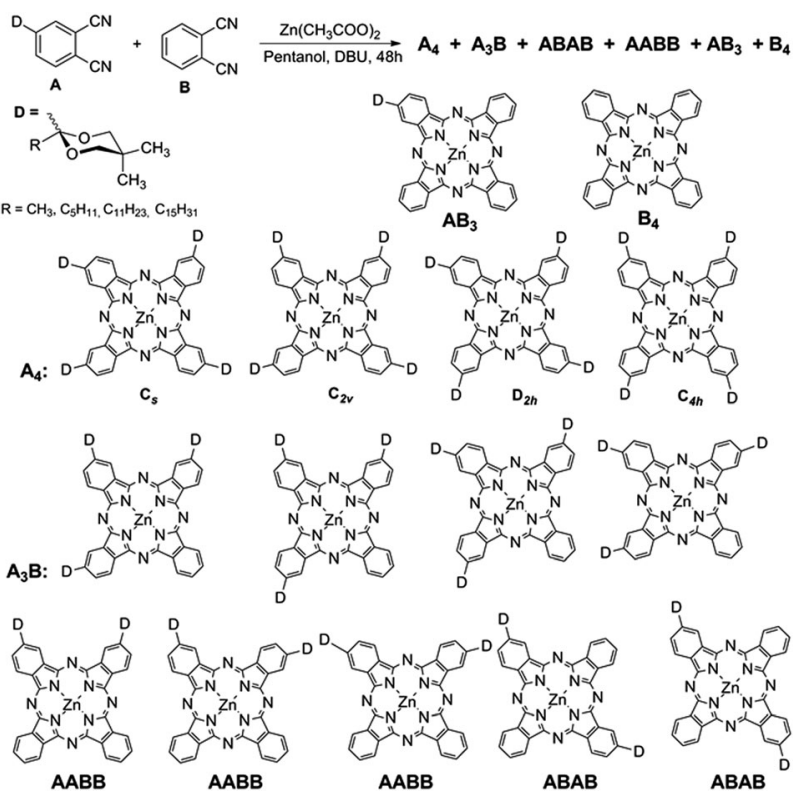

Scheme 2 Mixed condensation of two different phthalodinitriles $\mathbf{A}$ and $\mathbf{B}$. D stands for the 1,3-dioxolane protecting group which after removal gives the acylated phthalocyanines with $\mathrm{RC}=\mathrm{O}$ being either acetyl, hexanoyl, lauroyl or palmitoyl residues. 
detective-like investigations of such mixtures which upon careful assignments become "informed" libraries ${ }^{18}$ of Pc's (Fig. S12-S15, $\mathrm{ESI} \dagger$ ). We envisaged that the different regioisomers could have different self-assembly properties. Indeed, the UV-Vis spectra of the four $\mathbf{A}_{\mathbf{4}}$ isomers show Q-bands with different intensities (Fig. S13, ESI $\dagger$ ), and in future work, by combining with theoretical calculations, an assignment of the regioisomers will be attempted. Up-scaling of the HPLC-separations need to be performed in order to allow also NMR-based assignments.

In a truly biomimetic approach, we envisaged working with such mixtures because in the natural chlorosomes a similar heterogeneity was optimized by evolution. In the Cloroflexus aurantiacus antennas, the same BChl $c$ core chromophore is esterified at the $17^{3}$ position by various long chain aliphatic alcohols such as cetyl, stearyl, oleyl, geranyl-geranyl etc. ${ }^{19}$ in various proportions depending on the habitat. On the contrary, in Chlorobaculum tepidum chlorosomes, the heterogeneity is ensured by different $\mathrm{R}^{8}$ and $\mathrm{R}^{12}$ substituents while the sole esterifying alcohol is farnesol (Fig. 1a). While $R^{8}$ changes from ethyl, propyl to isobutyl, $\mathrm{R}^{12}$ can be methyl or ethyl. Depending on the illumination conditions and, most intriguingly, on the stereochemistry of the 3-hydroxyethyl group involved in the self-assembly algorithm, the composition of these chlorosomal BChl homologues can vary significantly. We have also shown previously that the isolated pure enantiomers self-assemble less readily than their scalemic mixtures or racemates. ${ }^{20}$

With this heterogeneity in mind we performed a sodium borohydride reduction by using it in substoichiometric amounts on the mixture of Pc's so that only partial reduction of the acyl groups occurred to the corresponding racemic 2-hydroxyalkanols. Gratifyingly, we could put into evidence by HR-MS a compound whose mass corresponds to the monoreduced diacyl derivative. Although the exact desired geometry as shown in Fig. 1c, is not yet ascertained (as both AABB and the targeted ABAB types have the same mass), the fact that upon self-assembly, the typical chlorosomal red-shift and line broadening occurs, indicates that the self-assembly algorithm operates correctly and that regioisomers which do not have the ideal geometry, leading to possible defects, will be reversibly disassembled from the final structure. This self-healing is typical for supramolecular assemblies in a thermodynamically driven process. We can thus preferentially select the species which will self-assemble effectively, and after repeated cycles of centrifugations and re-suspensions, the Pc's that do not self-assemble are simply discarded with the supernatant.

In summary, we firstly optimized a way to synthesize for the first time highly soluble acylphthalocyanines by employing carbonylprotected groups, and secondly, due to the recognition ability of the carbonyl groups, the central zinc atom and the solubilizing alkyl chains, these could self-assemble in the same way as the natural BChl's. The supramolecular self-assemblies of these acylphthalocyanines in $n$-heptane show a very broad near-infrared Q-band absorption with an onset at over $900 \mathrm{~nm}$.

H.-G. Jin gratefully acknowledges his $\mathrm{PhD}$ fellowship from the China Scholarship Council. Dr Valèrie Monnier and Christophe Chendo are thanked for the high-resolution mass spectra recorded at the Spectropole in Marseille. Prof. Harry L. Anderson is thanked for helpful discussions on cooperative aggregation mechanisms.

\section{Notes and references}

1 (a) J.-M. Lehn, Science, 2002, 295, 2400; (b) A. C. Grimsdale and K. Müllen, Angew. Chem., Int. Ed., 2005, 44, 5592; (c) P. Kambhampati, Acc. Chem. Res., 2011, 44, 1; (d) J. Rawson, A. C. Stuart, W. You and M. J. Therien, J. Am. Chem. Soc., 2014, 136, 17561.

2 (a) G. McDermott, S. M. Prince, A. A. Freer, A. M. HawthornthwaiteLawless, M. Z. Papiz, R. J. Cogdell and N. W. Isaacs, Nature, 1995, 374, 517; (b) M. Z. Papiz, S. M. Prince, T. Howard, R. J. Cogdell and N. W. Isaacs, J. Mol. Biol., 2003, 326, 1523; (c) A. W. Roszak, T. D. Howard, J. Southall, A. T. Gardiner, C. J. Law, N. W. Isaacs and R. J. Cogdell, Science, 2003, 302, 1969.

3 (a) Phthalocyanines - Properties and Applications, ed. C. C. Leznoff and A. P. B. Lever, VCH, New York, vol. 1, 1989, vol. 2, 1992; vol. 3,1993; vol. 4, 1996; (b) Porphyrin Handbook, ed. K. M. Kadish, K. M. Smith and R. Guilard, Academic Press, San Diego, vol. 15-20, 2003; (c) Handbook of Porphyrin Science with Applications to Chemistry, Physics, Materials Science, Engineering, Biology and Medicine, K. M. Kadish, K. M. Smith and R. Guilard, World Scientific, Singapore, vol. 1-35, 2010-2015; (d) M. V. Martinez-Diaz, G. de la Torre and T. Torres, Chem. Commun., 2010, 46, 7090; (e) M. G. Walter, A. B. Rudine and C. C. Wamser, J. Porphyrins Phthalocyanines, 2010, 14, 759; $(f)$ J. Mack and N. Kobayashi, Chem. Rev., 2011, 111, 281; $(g)$ G. de la Torre, G. Bottari, M. Sekita, A. Hausmann, D. M. Guldi and T. Torres, Chem. Soc. Rev., 2013, 42, 8049.

4 (a) M. E. Ragoussi, J. J. Cid, J. H. Yum, G. de la Torre, D. Di Censo, M. Grätzel, M. K. Nazeeruddin and T. Torres, Angew. Chem., Int. Ed., 2012, 51, 4375; (b) T. Ikeuchi, H. Nomoto, N. Masaki, M. J. Griffith, S. Mori and M. Kimura, Chem. Commun., 2014, 50, 1941.

5 (a) J. A. A. W. Elemans, R. van Hameren, R. J. M. Nolte and A. E. Rowan, Adv. Mater., 2006, 18, 1251; (b) K. Kameyama, M. Morisue, A. Satake and Y. Kobuke, Angew. Chem., Int. Ed., 2005, 44, 4763.

6 (a) J. Overmann, H. Cypionka and N. Pfennig, Limnol. Oceanogr., 1992, 37, 150; (b) A. K. Manske, J. Glaeser, M. M. M. Kuypers and J. Overmann, Appl. Environ. Microbiol., 2005, 71, 8049.

7 (a) M. I. Bystrova, I. N. Mal'gosheva and A. A. Krasnovskii, Mol. Biol., 1979, 13, 440; (b) K. M. Smith, L. A. Kehres and J. Fajer, J. Am. Chem. Soc., 1983, 99, 1387.

8 (a) T. S. Balaban, A. D. Bhise, M. Fischer, M. Linke-Schaetzel, C. Roussel and N. Vanthuyne, Angew. Chem., Int. Ed., 2003, 42, 2140; (b) T. S. Balaban, M. Linke-Schaetzel, A. D. Bhise, N. Vanthuyne and C. Roussel, Eur. J. Org. Chem., 2004, 3919.

9 (a) M. C. Balaban and T. S. Balaban, J. Porphyrins Phthalocyanines, 2007, 11, 277; (b) J. Szmytkowski, J. Conradt, H. Kuhn, C. M. Reddy, M. C. Balaban, T. S. Balaban and H. Kalt, J. Phys. Chem. C, 2011, 115, 8832.

10 T. Jochum, C. M. Reddy, A. Eichhofer, G. Buth, J. Szmytkowski, H. Kalt, D. Moss and T. S. Balaban, Proc. Natl. Acad. Sci. U. S. A., 2008, 105, 12736.

11 (a) E. E. Jelley, Nature, 1936, 138, 1009-1010; (b) G. Z. Scheibe, Angew. Chem., 1936, 49, 563; (c) G. Scheibe, A. Mareis and H. Ecker, Naturwissenschaften, 1937, 25, 474; (d) A. Mishra, R. K. Behera, P. K. Behera, B. K. Mishra and G. B. Behera, Chem. Rev., 2000, 100, 1973; (e) F. Würthner, T. E. Kaiser and C. R. Saha-Möller, Angew. Chem., Int. Ed., 2011, 50, 3376.

12 (a) M. C. Balaban and T. S. Balaban, US0054166 A1, 2011; (b) M. C. Balaban and T. S. Balaban, EP2292623A1, 2011.

13 T. S. Balaban, J. Leitich, A. R. Holzwarth and K. Schaffner, J. Phys. Chem. B, 2000, 104, 1362.

14 (a) M. Sommerauer, C. Rager and M. Hanack, J. Am. Chem. Soc., 1996, 118, 10085; (b) S. Rodríguez-Morgade and M. Hanack, Chem. - Eur. J., 1997, 3, 1042; (c) B. Görlach, M. Dachtler, T. Glaser, K. Albert and M. Hanack, Chem. - Eur. J., 2001, 7, 2459.

15 C. C. Leznoff, C. R. McArthur and Y. N. Qin, Can. J. Chem., 1993, 71, 1319. 16 K. Arnold, T. S. Balaban, M. N. Blom, O. T. Ehrler, S. Gilb, O. Hampe, J. E. van Lier, J. M. Weber and M. M. Kappes, J. Phys. Chem. A, 2003, 107, 794.

17 Z. Jiang, W. He, H. Yao, J. Wang, N. Chen and J. Huang, J. Porphyrins Phthalocyanines, 2011, 15, 140.

18 O. Ramström and J.-M. Lehn, Nat. Rev. Drug Discovery, 2002, 1, 26.

19 F. Fages, N. Griebenow, K. Griebenow, A. R. Holzwarth and K. Shaffner, J. Chem. Soc., Perkin Trans. 1, 1990, 2791.

20 T. S. Balaban, A. D. Bhise, G. Bringmann, J. Bürck, C. Chappaz-Gillot, A. Eichhöfer, D. Fenske, D. C. G. Götz, M. Knauer, T. Mizoguchi, D. Mössinger, H. Rösner, C. Roussel, M. Schraut, H. Tamiaki and N. Vanthuyne, J. Am. Chem. Soc., 2009, 131, 14480. 